\title{
Backward Ricci flow on locally homogeneous 3-manifolds
}

\author{
Xiaodong Cao and Laurent Saloff-Coste
}

\begin{abstract}
In this paper, we study the backward Ricci flow on locally homogeneous 3-manifolds. We describe the long time behavior and show that, typically and after a proper re-scaling, there is convergence to a sub-Riemannian geometry. A similar behavior was observed by the authors in the case of the cross curvature flow.
\end{abstract}

\section{Introduction}

\subsection{The Ricci flow}

In [6], Isenberg and Jackson studied the Ricci flow on homogeneous 3-manifolds. As homogeneous 3-manifolds are the models and building blocks of the geometrization of 3-manifolds, it is natural and important to study the behavior of various geometric flows in this basic case. See [2, Chapter 1]. Further studies are in $[5,7,8]$.

For obvious reasons, works have focused on the forward behavior of the Ricci flow although, in the homogeneous case, the flow reduces to a system of ordinary differential equations and there is no obstruction to the study of the backward flow. In $[3,4]$, the authors studied the forward and backward limits of the cross curvature flow on homogeneous 3-manifolds. Indeed, in the case of the cross curvature flow it is not entirely clear which direction is more natural. The results obtained in [4] suggest that the backward behavior of the Ricci flow should be studied as well and this is the subject of this paper.

Recall that the Ricci flow on a manifold is a flow of Riemannian metric $g(t)$ satisfying the equation

$$
\frac{\partial g}{\partial t}=-2 \mathrm{Rc}, \quad g(0)=g_{0}
$$

where Rc denotes the Ricci curvature tensor (in this instance, the Ricci curvature tensor of the metric $g(t)$ ). This can be normalized in various ways 
by setting $\tilde{g}(\tilde{t})=\psi(t) g(t), \tilde{t}=\int_{0}^{t} \psi(s) d s$. Setting $\tilde{\psi}(\tilde{t})=\psi(t)$, we obtain

$$
\frac{\partial \tilde{g}}{\partial \tilde{t}}=-2 \widetilde{\mathrm{Rc}}+\left(\frac{\partial \ln \tilde{\psi}}{\partial \tilde{t}}\right) \tilde{g}, \quad \tilde{g}(0)=g_{0}
$$

For compact manifolds, the customary normalization uses $\frac{1}{\psi} \frac{\partial \psi}{\partial t}=\frac{2 r}{3}$, where $r$ is the average of the scalar curvature $R$, in which case, $\frac{1}{\tilde{\psi}} \frac{\partial \tilde{\psi}}{\partial \tilde{t}}=\frac{2 \tilde{r}}{3}$. This normalization keeps the volume constant under the flow. In the case of the locally homogeneous manifolds, we can use this normalization even in the non-compact case since the scalar curvature is constant. Hence, following [6], we will study the flow

$$
\frac{\partial g}{\partial t}=-2 \mathrm{Rc}+\frac{2}{3} \operatorname{Rg}, \quad g(0)=g_{0}
$$

\subsection{The backward behavior of the Ricci flow}

There are 9 types of locally homogeneous 3-manifolds and these are split into two families. The first family contains the manifolds covered by the hyperbolic 3 -space $H_{3}$, and the product geometries of type $H_{2} \times \mathbb{R}$ and $\mathbb{S}_{2} \times \mathbb{R}$. The second family corresponds to those geometries whose universal cover is a group itself. They are: $\mathbb{R}^{3}, \mathrm{SU}(2, \mathbb{R}) ; \mathrm{SL}(2, \mathbb{R}) ; E(1,1)=$ Sol, i.e., the group of isometries of a flat Lorentz plane; $\widetilde{E(2)}$, the universal cover of group of isometries of the plane; the Heisenberg group. This second family is referred to as the Bianchi case (see [6]). In the Bianchi case, given a metric $g_{0}$, Milnor [9] provides a frame $\left(f_{1}, f_{2}, f_{3}\right)$ in which both the metric and the Ricci tensors are diagonalized. As this property is preserved by the Ricci flow, writing

$$
g=A f^{1} \otimes f^{1}+B f^{2} \otimes f^{2}+C f^{3} \otimes f^{3}
$$

the Ricci flow becomes an ODE system in $(A, B, C)$. Furthermore, Milnor's paper [9] provides the computation of the Ricci tensor in each case so that the ODE system in question can be written down explicitly. The simplest non-trivial case is the Heisenberg group. Given a metric $g_{0}$ on the Heisenberg group (or on a 3-manifold of Heisenberg type), we fix a Milnor frame $\left\{f_{i}\right\}_{1}^{3}$ such that $\left[f_{2}, f_{3}\right]=2 f_{1},\left[f_{3}, f_{1}\right]=0$ and $\left[f_{1}, f_{2}\right]=0$. Using [9], the ODE 
system for the normalized Ricci flow is given by

$$
\left\{\begin{array}{l}
\frac{d A}{d t}=-\frac{16}{3} \frac{A^{3}}{A_{0} B_{0} C_{0}} \\
\frac{d B}{d t}=+\frac{8}{3} \frac{A^{2} B}{A_{0} B_{0} C_{0}} \\
\frac{d C}{d t}=+\frac{8}{3} \frac{A^{2} C}{A_{0} B_{0} C_{0}}
\end{array}\right.
$$

where we used the fact that, under (1.1), $A B C=A_{0} B_{0} C_{0}$. Let $R_{0}=-\frac{2 A_{0}}{B_{0} C_{0}}$ $<0$ be the initial scalar curvature. Then (1.2) admits a completely explicit maximal solution defined on $\left(3 /\left(16 R_{0}\right),+\infty\right)$ and given by

$$
\left\{\begin{array}{l}
A(t)=A_{0}\left(1-(16 / 3) R_{0} t\right)^{-1 / 2} \\
B(t)=B_{0}\left(1-(16 / 3) R_{0} t\right)^{1 / 4} \\
C(t)=C_{0}\left(1-(16 / 3) R_{0} t\right)^{1 / 4}
\end{array}\right.
$$

Observe that the metric $\bar{g}(t)=\left(C_{0} / C(t)\right) g(t)$ converges when $t$ tends to $3 /\left(16 R_{0}\right)=-T_{b}$ to

$$
\infty f^{1} \otimes f^{1}+B_{0} f^{2} \otimes f^{2}+C_{0} f^{3} \otimes f^{3}
$$

which can be interpreted as describing a sub-Riemannian geometry on the Heisenberg group. The point of this paper is to show that this behavior in the backward direction is typical for all locally homogeneous manifolds corresponding to the Bianchi cases described above except for those corresponding to the trivial case $\mathbb{R}^{3}$.

Theorem 1.1. Let $\left(M, g_{0}\right)$ be a locally homogeneous 3-manifold with universal cover $\mathrm{SU}(2, \mathbb{R}), \widehat{\mathrm{SL}(2, \mathbb{R})}, E(1,1)=\mathrm{Sol}, \widetilde{E(2)}$ or the Heisenberg group. Let $g(t),\left(-T_{b}, T_{f}\right)$ be a maximal solution of the normalized Ricci flow (1.1). Let $d(t)$ be the associated distance function on $M$. Assume $g_{0}$ is generic among all locally homogeneous metric on $M$. Then $T_{b}$ is finite and there exists a function $r(t):\left(-T_{b}, 0\right] \rightarrow(0, \infty)$ such that, as $t$ tends to $-T_{b}$, the metric spaces $(M, r(t) d(t))$ converge uniformly to a sub-Riemannian space $\left(M, d_{b}\right)$ whose tangent cone at any point is the Heisenberg group equipped with its natural sub-Riemannian metric.

By definition, the uniform convergence of metric spaces $\left(M, d_{t}\right)$ to $(M, d)$ means the uniform convergence over compact sets of $(x, y) \rightarrow d_{t}(x, y)$ to $(x, y) \rightarrow d(x, y)$. 
The present paper proves this theorem in all cases except $\widehat{\mathrm{SL}(2, \mathbb{R})}$. For manifolds covered by $\widehat{\mathrm{SL}(2, \mathbb{R})}$, we prove the result under the additional assumption that there exists a time $t_{0}$ such that either $A\left(t_{0}\right) \geq \max \left\{B\left(t_{0}\right)\right.$, $\left.C\left(t_{0}\right)\right\}$ or $A\left(t_{0}\right) \leq\left|B\left(t_{0}\right)-C\left(t_{0}\right)\right|$. In the paper [1], we show that this condition is always satisfied except for a hypersurface of initial conditions.

The proof of this theorem proceeds by inspection of the different cases. It would be more elegant to have an argument covering all cases at once. However, the existence of exceptional sets of initial conditions for which the general result fails indicates that it is unlikely that such treatment is possible. Indeed, the exceptional sets of initial conditions are very much case dependent, see the more precise statements in the different sections below.

The results obtained in each of the different cases are more precise than stated in Theorem 1.1. They describe the asymptotic behavior of each of the metric components in a fixed Milnor frame. This is useful in exploring the Ricci flow on homogeneous 3-manifolds under more sophisticated scaling procedures. See $[5,7,8]$.

Together, the study of the forward normalized Ricci flow (see [6-8]) and this paper, give a description of the asymptotic behaviors of the Ricci flow on homogeneous 3-manifolds for both the forward and backward directions. For instance, the solutions of the forward normalized Ricci flow always exist for all (positive) time in the Bianchi classes [6].

\subsection{Sub-Riemannian geometries}

Our main result, Theorem 1.1, refers to the notion of sub-Riemannian geometry, a term that we now explain in the present context. The typical behavior (possibly after some re-scaling) of the evolving metric

$$
g=A f^{1} \otimes f^{1}+B f^{2} \otimes f^{2}+C f^{3} \otimes f^{3}
$$

at the end points of a maximal existence interval is that some of the coefficients $A, B, C$ either vanish or tend to $\infty$. When a coefficient vanishes and the manifold is compact, the phenomenon can be interpreted as a dimensional collapse. Naively, at least one direction disappears. To interpret the situation when a coefficient tends to infinity, it is useful to look at the dual tensor

$$
Q=A^{-1} f_{1} \otimes f_{1}+B^{-1} f_{2} \otimes f_{2}+C^{-1} f_{3} \otimes f_{3}
$$


defined on the co-tangent bundle. Suppose that $A$ tends to infinity whereas $B, C$ have finite limits $B_{*}, C_{*}$. Then the tensor $Q$ tends to

$$
Q_{*}=B_{*}^{-1} f_{2} \otimes f_{2}+C_{*}^{-1} f_{3} \otimes f_{3}
$$

If it turns out that $\left[f_{2}, f_{3}\right]=2 \epsilon_{1} f_{1}$ with $\epsilon_{1} \neq 0$, then the tensor $Q_{*}$ induces a natural distance function $d_{*}$ on the underlying manifold $M$. This distance can be computed by minimizing the length of the so-called horizontal curves, i.e., those curves that stay tangent to the linear span of $f_{2}, f_{3}$. The associated "geometry" is called a sub-Riemannian geometry. See [10] for a book length introduction to sub-Riemannian geometry and [4] for some details directly relevant to the present situation. Let us note here that the convergence $Q \rightarrow Q_{*}$ translates quite easily in the uniform convergence over compact sets on $M \times M$ of the associated distance functions. This explains the conclusion of Theorem 1.1.

\subsection{The normalized backward Ricci flow}

In order to study the backward behavior of the Ricci flow, it is convenient to reverse time and consider the solution of the positive normalized Ricci flow equation

$$
\frac{\partial g}{\partial t}=2 \mathrm{Rc}-\frac{2}{3} \mathrm{Rg}, \quad g(0)=g_{0}
$$

We let $T_{+} \in[0,+\infty]$ be the maximal existence time for this equation. The rest of this paper is devoted to the asymptotic properties of this flow when $t \rightarrow T_{+}$in the case of SU(2), E $(1,1), \widetilde{\mathrm{E}(2)}$ and $\widehat{\mathrm{SL}(2, \mathbb{R})}$. This includes determining whether $T_{+}$is finite or infinite. The results are stated explicitly for the flow on each of these groups but, in each case, it holds in the same form on any locally homogeneous 3 -manifold covered by the corresponding group. In each case, we write the solution of (1.3) in the form

$$
g=A f^{1} \otimes f^{1}+B f^{2} \otimes f^{2}+C f^{3} \otimes f^{3}
$$

in a Milnor frame $\left(f_{1}, f_{2}, f_{3}\right)$ for $g_{0}$. Under (1.3), $A B C=A_{0} B_{0} C_{0}$ is a constant. In the rest of this paper, we assume the normalization $A_{0} B_{0} C_{0}=4$. This choice is made so that the ODE systems are the same as in [6], despite the fact that the frame we use here have a different normalization than those used in [6]. 
If $A, B, C$ is the solution under $A_{0} B_{0} C_{0}=4$ and $\tilde{A}, \tilde{B}, \tilde{C}$ is the solution with $\widetilde{A_{0}}=\lambda A_{0}, \widetilde{B_{0}}=\lambda B_{0}, \widetilde{C_{0}}=\lambda C_{0}$, then $\tilde{A}(t)=\lambda A(t / \lambda), \tilde{B}(t)=\lambda B(t / \lambda)$ and $\tilde{C}(t)=\lambda C(t / \lambda)$.

\section{The normalized positive Ricci flow on $\mathrm{SU}(2)$}

Given a metric $g_{0}$ on $\mathrm{SU}(2)$, we fix a Milnor frame such that $\left[f_{i}, f_{j}\right]=2 f_{k}$ for all cyclic permutations of the indices. This section is devoted to the proof of the following result.

Theorem 2.1. Let $g_{0}$ be a homogeneous metric on $\mathrm{SU}(2)$ with associated Milnor frame $\left(f_{1}, f_{2}, f_{3}\right)$ and $g_{0}=A_{0} f^{1} \otimes f^{1}+B_{0} f^{2} \otimes f^{2}+C_{0} f^{3} \otimes f^{3}$ with $A_{0} B_{0} C_{0}=4$. Let $g(t)=A(t) f^{1} \otimes f^{1}+B(t) f^{2} \otimes f^{2}+C(t) f^{3} \otimes f^{3}, t \in$ $\left[0, T_{+}\right)$be the maximal forward solution of the positive normalized Ricci flow (1.3) with $g(0)=g_{0}$. Assume that $A_{0} \geq B_{0} \geq C_{0}$.

(1) If $A_{0}=B_{0}=C_{0}$ then $T_{+}=\infty$ and $g(t)=g_{0}, t \in[0, \infty)$.

(2) If $A_{0}=B_{0}>C_{0}$ then $T_{+}=\infty, A=B>C$ and, as $t$ tends to infinity, $A \sim \frac{8}{3} t, C \sim \frac{9}{16} t^{-2}$.

(3) If $A_{0}>B_{0} \geq C_{0}$ then $T_{+}$is finite, $A>B \geq C$ and there are constants $\eta_{1}, \eta_{2} \in(0, \infty)$ such that

$$
A \sim \frac{\sqrt{6}}{4}\left(T_{+}-t\right)^{-1 / 2}, \quad B \sim \eta_{1}\left(T_{+}-t\right)^{1 / 4}, \quad C \sim \eta_{2}\left(T_{+}-t\right)^{1 / 4}
$$

as $t$ tends to $T_{+}$.

Let $d(t)$ be the distance function associated to $\left(B_{0} / B(t)\right) g(t)$. In case (3), the metric space $(\mathrm{SU}(2), d(t))$ converges uniformly as $t \rightarrow T_{+}$towards the sub-Riemanninan metric space $\left(\mathrm{SU}(2), d_{*}\right)$ where $d_{*}$ is the sub-Riemannian distance associated with

$$
Q_{*}=B_{0}^{-1} f_{2} \otimes f_{2}+\eta_{1} \eta_{2}^{-1} B_{0}^{-1} f_{3} \otimes f_{3} .
$$

Remark 2.1. Consider a maximal solution

$$
g_{f}(t)=A(t) f^{1} \otimes f^{1}+B(t) f^{2} \otimes f^{2}+C(t) f^{3} \otimes f^{3}, \quad t \in\left(-T_{+}, \infty\right)
$$

of the forward normalized Ricci flow (1.1). Let $\bar{g}(t)=\left(B_{0} / B(t)\right) g_{f}(t)$. Isenberg and Jackson [6] shows that $A-C \leq\left(A_{0}-C_{0}\right) e^{-2 C_{0}^{2} t}, \forall t \geq 0$, if $A_{0} \geq$ $B_{0} \geq C_{0}$ (this order is preserved by the flow). Hence, in the forward 
direction, $\bar{g}(t)$ converges exponentially fast to the round metric whereas Theorem 2.1 describes the backward behavior. In the generic case $A_{0}>$ $B_{0} \geq C_{0}, \bar{g}(t)$ converges to a sub-Riemannian metric as $t \rightarrow-T_{+}$.

The sectional curvatures are (see, e.g., [2, p. 12])

$$
\begin{aligned}
& K\left(f_{2} \wedge f_{3}\right)=\frac{(B-C)^{2}}{A B C}-\frac{3 A}{B C}+\frac{2}{B}+\frac{2}{C} \\
& K\left(f_{3} \wedge f_{1}\right)=\frac{(C-A)^{2}}{A B C}-\frac{3 B}{C A}+\frac{2}{A}+\frac{2}{C} \\
& K\left(f_{1} \wedge f_{2}\right)=\frac{(A-B)^{2}}{A B C}-\frac{3 C}{A B}+\frac{2}{A}+\frac{2}{B} .
\end{aligned}
$$

From the sectional curvatures given above, we easily obtain the ODEs corresponding to the flow, under the normalization $A B C=4$, namely,

$$
\left\{\begin{array}{l}
\frac{d A}{d t}=-\frac{2}{3} A\left[-A(2 A-B-C)+(B-C)^{2}\right] \\
\frac{d B}{d t}=-\frac{2}{3} B\left[-B(2 B-A-C)+(A-C)^{2}\right] \\
\frac{d C}{d t}=-\frac{2}{3} C\left[-C(2 C-A-B)+(A-B)^{2}\right]
\end{array}\right.
$$

Without loss of generality, we may assume that $A_{0} \geq B_{0} \geq C_{0}$. As

$$
\begin{aligned}
& \frac{d}{d t}(A-C)=\frac{2}{3}(A-C)\left[2 A^{2}+2 A C+2 C^{2}-(A+B+C) B\right] \\
& \frac{d}{d t}(A-B)=\frac{2}{3}(A-B)\left[2 A^{2}+2 A B+2 B^{2}-(A+B+C) C\right] \\
& \frac{d}{d t}(B-C)=\frac{2}{3}(B-C)\left[2 B^{2}+2 B C+2 C^{2}-(A+B+C) A\right]
\end{aligned}
$$

it is easy to see that $A \geq B \geq C$ is preserved along the flow. This yields the following lemma.

Lemma 2.1. Assume that $A_{0} \geq B_{0} \geq C_{0}$. Then $A, A-B$ and $A-C$ are all non-decreasing along the flow and $C$ is non-increasing.

We now consider three cases. The first case is when $A_{0}=B_{0}=C_{0}$. Then $A(t)=B(t)=C(t)=A_{0}$ and the solution exists for all time. 
The second case is when $A_{0}=B_{0}>C_{0}$. Then $A(t)=B(t)$ as long as the solution exists and we have

$$
\left\{\begin{array}{l}
\frac{d A}{d t}=\frac{2}{3} A C(A-C) \\
\frac{d C}{d t}=-\frac{4}{3} C^{2}(A-C) .
\end{array}\right.
$$

In this case, $A$ is increasing, $C$ is decreasing and $A^{2} C=A_{0}^{2} C_{0}$ along the flow.

Lemma 2.2. If $A_{0}=B_{0}>C_{0}$, then $T_{+}=\infty, A \sim \frac{8}{3} t$ and $C \sim \frac{9}{16} t^{-2}$ as $t$ tends to infinity.

Proof. Since $\frac{d A}{d t}=\frac{2}{3} A_{0}^{2} C_{0}-\frac{2}{3} A C^{2}$, if $T_{+}<\infty$, then $\lim _{T_{+}} A<\infty$, and $\lim _{T_{+}}$ $C>0$. This contradicts the assumption that $T_{+}$is the maximal existence time. Hence $T_{+}=\infty$. As $A$ is increasing, $C$ is decreasing and $A^{2} C$ constant, it follows from (2.5) that $\lim _{\infty} A=\infty$, and thus $\lim _{\infty} C=0$. Moreover, $\lim _{\infty} A C^{2}=0$. Now the asymptotic for $A$ and $C$ follows from (2.5) which yields $\frac{d}{d t} A \sim \frac{2}{3} A_{0}^{2} C_{0}$, and $A^{2} C=A_{0}^{2} C_{0}=4$.

We now focus on the third case, the generic case.

Lemma 2.3. Assume that $A_{0}>B_{0} \geq C_{0}$. Then $T_{+}<\infty$.

Proof. Assume that $T_{+}=\infty$. We have

$$
\frac{d}{d t} A=\frac{2}{3} A\left[A(A-B)+A(A-C)-(B-C)^{2}\right] \geq \frac{2}{3} A^{2}(A-B)
$$

and

$$
\frac{d}{d t} C<-\frac{2}{3} C(A-B)^{2} .
$$

Since, by Lemma 2.1, both $A$ and $A-B$ are non-decreasing, it follows that $\lim _{\infty} A=\infty, \lim _{\infty} C=0$. Now, (2.3) implies that

$$
\frac{d}{d t} \ln (A-B) \geq \frac{2}{3}\left(2 A^{2}+B^{2}\right)
$$

hence $\lim _{\infty}(A-B)=\infty$. Since

$$
\frac{d}{d t} \ln B=-\frac{2}{3}\left[B(A-B)+(A-B)^{2}+2(A-B)(B-C)-C(B-C)\right]
$$


this shows that $B$ is non-increasing for $t$ large enough, hence bounded. So, we have

$$
\frac{d A}{d t} \sim \frac{4}{3} A^{3}
$$

But this shows that there exists a finite time $T_{0}$, such that $\lim _{T_{0}} A=\infty$, this contradicts our assumption that $T_{+}=\infty$.

Lemma 2.4. Assume $A_{0}>B_{0} \geq C_{0}$. Then $\lim _{T_{+}} A=\infty$, $\lim _{T_{+}} B=\lim _{T_{+}}$ $C=0$.

Proof. Assume that $\lim _{T_{+}} C>0$. As $A>B \geq C$ and that $T_{+}$is finite, we must have $\lim _{T_{+}} A=\infty$. We have

$$
\frac{d}{d t} \ln A<\frac{4}{3} A^{2} \leq \frac{d}{d t} \ln (A-B) .
$$

It follows that $\lim _{T_{+}}(A-B)=\infty$. By (2.6), $B$ is non-increasing for $t$ close to $T_{+}$and hence bounded from above. This shows that $\frac{d}{d t} A \sim \frac{4}{3} A^{3}$ and thus that $A^{-2} \sim \frac{8}{3}\left(T_{+}-t\right)$. Hence

$$
\frac{d}{d t} \ln C \sim-\frac{1}{4}\left(T_{+}-t\right)^{-1}
$$

This contradicts $\lim _{T_{+}} C>0$ and we conclude that $\lim _{T_{+}} C=0$.

Now by (2.6) we can see that $B$ is bounded from above. So, if $\lim _{T_{+}}$ $A<\infty$ then $\frac{d}{d t} C \sim-\eta C$, for some constant $\eta \in(0, \infty)$. This contradicts $\lim _{T_{+}}$ $C=0$. So we conclude that $\lim _{T_{+}} A=\infty$.

To show that $\lim _{T_{+}} B=0$, notice that (2.6) implies that $B$ is nonincreasing for $t$ close enough to $T_{+}$. As

$$
\frac{d}{d t} \ln \left(A B^{2}\right)=2(B-C)(B+C-A)
$$

we obtain that $A B^{2}$ is bounded from above on $\left[0, T_{+}\right)$, hence $\lim _{T_{+}} B=0$.

Lemma 2.5. Assume that $A_{0}>B_{0} \geq C_{0}$, then there exist $\eta_{1}, \eta_{2} \in(0, \infty)$, such that

$$
A \sim \frac{\sqrt{6}}{4}\left(T_{+}-t\right)^{-1 / 2}, \quad B \sim \eta_{1}\left(T_{+}-t\right)^{1 / 4}, \quad C \sim \eta_{2}\left(T_{+}-t\right)^{1 / 4} .
$$


Proof. The first statement follows directly from

$$
\frac{d}{d t} A \sim \frac{4}{3} A^{3}
$$

To obtain the asymptotic behavior for $B, C$, notice that

$$
\frac{d}{d t} \ln \left(A C^{2}\right)=2(B-C)(A-B-C), \frac{d}{d t} \ln \left(A B^{2}\right)=2(B-C)(B+C-A) .
$$

Since $\lim _{T_{+}} A=\infty$ and $\lim _{T_{+}} B=\lim _{T_{+}} C=0$, we have $\lim _{T_{+}}(A-B-$ $C)=\infty$. Hence, the equations above imply that $A B^{2}$ is non-increasing and $A C^{2}$ is non-decreasing for $t$ close to $T_{+}$. But $B \geq C$, so

$$
0<\lim _{T_{+}} A C^{2} \leq \lim _{T_{+}} A B^{2}<\infty .
$$

It follows that $\lim _{T_{+}} B \sim \eta_{1}\left(T_{+}-t\right)^{1 / 4}$ and $\lim _{T_{+}} C \sim \eta_{2}\left(T_{+}-t\right)^{1 / 4}$.

This finishes the proof of Theorem 2.1.

\section{The normalized positive Ricci flow on $E(1,1)$ (Sol geometry)}

Given a metric $g_{0}$ on $E(1,1)$, we fix a Milnor frame such that $\left[f_{2}, f_{3}\right]=$ $2 f_{1},\left[f_{3}, f_{1}\right]=0,\left[f_{1}, f_{2}\right]=-2 f_{3}$. This section is devoted to the proof of the following result.

Theorem 3.1. Let $g_{0}$ be a homogeneous metric on $E(1,1)$ with associated Milnor frame $\left(f_{1}, f_{2}, f_{3}\right)$ and $g_{0}=A_{0} f^{1} \otimes f^{1}+B_{0} f^{2} \otimes f^{2}+C_{0} f^{3} \otimes f^{3}$ with $A_{0} B_{0} C_{0}=4$. Let $g(t)=A(t) f^{1} \otimes f^{1}+B(t) f^{2} \otimes f^{2}+C(t) f^{3} \otimes f^{3}, t \in$ $\left[0, T_{+}\right)$be the maximal forward solution of the positive normalized Ricci flow (1.3) with $g(0)=g_{0}$. Assume that $A_{0} \geq C_{0}$.

(1) If $A_{0}=C_{0}$ then $T_{+}=\frac{3}{32} B_{0}$ and

$$
A(t)=C(t)=\frac{\sqrt{6}}{4}\left(T_{+}-t\right)^{-1 / 2}, \quad B(t)=\frac{32}{3}\left(T_{+}-t\right), \quad t \in\left[0, T_{+}\right) .
$$

(2) If $A_{0}>C_{0}$ then $T_{+}<\infty$ and, as $t$ tends to $T_{+}$, there are constants $\eta_{1}, \eta_{2} \in(0, \infty)$ such that

$$
A \sim \frac{\sqrt{6}}{4}\left(T_{+}-t\right)^{-1 / 2}, \quad B \sim \eta_{1}\left(T_{+}-t\right)^{1 / 4}, \quad C \sim \eta_{2}\left(T_{+}-t\right)^{1 / 4} .
$$


Let $d(t)$ be the distance function associated to $\left(B_{0} / B(t)\right) g(t)$. In case $(2)$, the metric space $(E(1,1), d(t))$ converges uniformly as $t \rightarrow T_{+}$towards the sub-Riemanninan metric space $\left(E(1,1), d_{*}\right)$ where $d_{*}$ is the sub-Riemannian distance associated with

$$
Q_{*}=B_{0}^{-1} f_{2} \otimes f_{2}+\eta_{1} \eta_{2}^{-1} B_{0}^{-1} f_{3} \otimes f_{3}
$$

Remark 3.1. For the forward normalized Ricci flow (1.1), Isenberg and Jackson [6] show that the solution exists for all time and presents a cigar degeneracy.

The sectional curvatures of $g(t)$ in the frame $\left(f_{i}\right)_{1}^{3}$ are:

$$
\begin{aligned}
& K\left(f_{2} \wedge f_{3}\right)=\frac{(A-C)^{2}-4 A^{2}}{A B C} \\
& K\left(f_{3} \wedge f_{1}\right)=\frac{(A+C)^{2}}{A B C} \\
& K\left(f_{1} \wedge f_{2}\right)=\frac{(A-C)^{2}-4 C^{2}}{A B C} .
\end{aligned}
$$

These yield the equations for the normalized positive Ricci flow on $E(1,1)$, under the normalization $A B C=4$, namely,

$$
\left\{\begin{array}{l}
\frac{d A}{d t}=\frac{2}{3} A\left(2 A^{2}+A C-C^{2}\right) \\
\frac{d B}{d t}=-\frac{2}{3} B(A+C)^{2} \\
\frac{d C}{d t}=\frac{2}{3} C\left(2 C^{2}+A C-A^{2}\right) .
\end{array}\right.
$$

Lemma 3.1. If $A_{0}=C_{0}$, then $T_{+}=\frac{3}{32} B_{0}<\infty$. Moreover $A(t)=C(t)=$ $\frac{\sqrt{6}}{4}\left(T_{+}-t\right)^{-1 / 2}$ and $B(t)=\frac{32}{3}\left(T_{+}-t\right)$, for $t \in\left[0, T_{+}\right)$.

Proof. It is easy to see that $A=C$ as long as the solution exists. As $\frac{d}{d t} A^{-2}=$ $-\frac{8}{3}$, we have $T_{+}<\infty$ and

$$
A=\frac{\sqrt{6}}{4}\left(T_{+}-t\right)^{-1 / 2}, \quad T_{+}=\frac{3}{8 A_{0}^{2}}=\frac{3}{32} B_{0} .
$$

Further, $\frac{d}{d t} B=-\frac{8}{3} B A^{2}$, so $B=\frac{32}{3}\left(T_{+}-t\right)$.

Without loss of generality, we assume that $A_{0}>C_{0}$. This implies that $A$ is increasing. Note that $B$ is always decreasing. 
Lemma 3.2. If $A_{0}>C_{0}$, then $T_{+}<\infty, \lim _{T_{+}} A=\infty$, and there exists $a$ time $t_{0}$ such that $A\left(t_{0}\right) \geq 2 C\left(t_{0}\right)$.

Proof. The fact that $T_{+}<\infty$ follows from $\frac{d}{d t} A>\frac{2}{3} A^{3}$. Now assume that $\lim _{T_{+}} A=A\left(T_{+}\right)<\infty$. Then, since

$$
\frac{d}{d t} \ln C=-\frac{2}{3}(A+C)(A-2 C)>-\frac{4}{3} A^{2}>-\frac{4}{3} A\left(T_{+}\right)^{2}
$$

and

$$
\frac{d}{d t} \ln B=-\frac{2}{3}(A+C)^{2}>\frac{8}{3} A^{2}>-\frac{8}{3} A\left(T_{+}\right)^{2}
$$

we get that $B \geq \lim _{T_{+}} B=B\left(T_{+}\right)>0$. Similarly, there exists some constant $\eta>0$ such that $C \in\left[\eta, A\left(T_{+}\right)\right]$. This contradicts the fact that the maximal existence time $T_{+}$is finite. Hence $\lim _{T_{+}} A=\infty$.

To prove the second statement, we assume that $C<A<2 C$ for all $t \in$ $\left[0, T_{+}\right)$. So we have $\lim _{T_{+}} C=\infty$. Since

$$
\frac{d}{d t} \ln (A / C)=2(A+C)(A-C)>0
$$

we see that $A / C$ is increasing, so $A / C>A_{0} / C_{0}$ and $A-C=A(1-C / A)>$ $\left(1-C_{0} / A_{0}\right) A$. Moreover, we have

$$
\left(1-C_{0} / A_{0}\right) \int_{0}^{T_{+}}(A+C) A<\int_{0}^{T_{+}}(A+C)(A-C)<\frac{1}{2} \ln 2 .
$$

Hence

$$
\int_{0}^{T_{+}}(A+C)(2 C-A)<\int_{0}^{T_{+}}(A+C) C<\int_{0}^{T_{+}}(A+C) A<\infty .
$$

This contradicts the fact that

$$
\frac{d}{d t} \ln C=\frac{2}{3}(A+C)(2 C-A) \text { and } \lim _{T_{+}} C=\infty .
$$

So there exists a time $t_{0}$ such that $A\left(t_{0}\right) \geq 2 C\left(t_{0}\right)$.

Lemma 3.3. Assume that $A_{0}>C_{0}$. There exist $\eta_{1}, \eta_{2} \in(0, \infty)$ such that, as $t$ tends to $T_{+}$, we have

$$
A \sim \frac{\sqrt{6}}{4}\left(T_{+}-t\right)^{-1 / 2}, \quad B \sim \eta_{1}\left(T_{+}-t\right)^{1 / 4}, \quad C \sim \eta_{2}\left(T_{+}-t\right)^{1 / 4}
$$


Proof. By Lemma 3.2, there is $t_{0}$ such that $A\left(t_{0}\right) \geq 2 C\left(t_{0}\right)$. As $\frac{d}{d t} \ln (A / C)=$ $2(A+C)(A-C)>0$, we conclude that $A(t) \geq 2 C(t)$ for $t \in\left[t_{0}, T_{+}\right)$. Hence $C$ is non-increasing on $\left[t_{0}, T_{+}\right)$. As

$$
\frac{d}{d t} \ln \left(A C^{3}\right)=\frac{2}{3}\left(-A^{2}+4 A C+5 C^{2}\right), \frac{d}{d t} \ln \left(A B^{2}\right)=-2 C(A+C)
$$

and $\lim _{T_{+}} A=\infty$, it follows that both $A C^{3}$ and $A B^{2}$ are bounded from above, hence $\lim _{T_{+}} B=\lim _{T_{+}} C=0$.

Next, we show that $\lim _{T_{+}} A B^{2}=\eta_{1}$ and $\lim _{T_{+}} A C^{2}=\eta_{2}$. Note that

$$
\frac{d}{d t} \ln \left(A C^{2}\right)=2 C(A+C) \text { and } \frac{d}{d t} \ln \left(A B^{2}\right)=-2 C(A+C) .
$$

Hence, it is enough to prove that $\int_{0}^{T_{+}} A C<\infty$. As $\frac{d}{d t} C \sim-\frac{2}{3} A^{2} C$, and $C>0$, we have

$$
\int_{0}^{T_{+}} A C<A_{0}^{-1} \int_{0}^{T_{+}} A^{2} C<\infty .
$$

Now, the lemma follows from $\frac{d}{d t} A \sim \frac{4}{3} A^{3}$.

This ends the proof of Theorem 3.1.

\section{The normalized positive Ricci flow on $\widetilde{E(2)}$}

Given a left-invariant metric $g_{0}$ on $\widetilde{E(2)}$, we fix a Milnor frame $\left\{f_{i}\right\}_{1}^{3}$ such that

$$
\left[f_{2}, f_{3}\right]=2 f_{1}, \quad\left[f_{3}, f_{1}\right]=2 f_{2}, \quad\left[f_{1}, f_{2}\right]=0 .
$$

The result in this case reads as follows.

Theorem 4.1. Let $g_{0}$ be a homogeneous metric on $\widetilde{E(2)}$ with associated Milnor frame $\left(f_{1}, f_{2}, f_{3}\right)$ and $g_{0}=A_{0} f^{1} \otimes f^{1}+B_{0} f^{2} \otimes f^{2}+C_{0} f^{3} \otimes f^{3}$ with $A_{0} B_{0} C_{0}=4$. Let $g(t)=A(t) f^{1} \otimes f^{1}+B(t) f^{2} \otimes f^{2}+C(t) f^{3} \otimes f^{3}, t \in\left[0, T_{+}\right)$ be the maximal forward solution of the positive normalized Ricci flow (1.3) with $g(0)=g_{0}$. Assume that $A_{0} \geq B_{0}$.

(1) If $A_{0}=B_{0}$ then $T_{+}=\infty$ and $g(t)=g_{0}$ on $[0, \infty)$.

(2) If $A_{0}>B_{0}$ then $T_{+}<\infty$ and, as $t$ tends to $T_{+}$, there are constants $\eta_{1}, \eta_{2} \in(0, \infty)$ such that

$$
A \sim \frac{\sqrt{6}}{4}\left(T_{+}-t\right)^{-1 / 2}, \quad B \sim \eta_{1}\left(T_{+}-t\right)^{1 / 4}, \quad C \sim \eta_{2}\left(T_{+}-t\right)^{1 / 4} .
$$


Let $d(t)$ be the distance function associated to $\left(B_{0} / B(t)\right) g(t)$. In case $(2)$, the metric space $(\widetilde{E(2)}, d(t))$ converges uniformly as $t \rightarrow T_{+}$towards the sub-Riemanninan metric space $\left(\widetilde{E(2)}, d_{*}\right)$ where $d_{*}$ is the sub-Riemannian distance associated with

$$
Q_{*}=B_{0}^{-1} f_{2} \otimes f_{2}+\eta_{1} \eta_{2}^{-1} B_{0}^{-1} f_{3} \otimes f_{3}
$$

Remark 4.1. Consider a maximal solution

$$
g_{f}(t)=A(t) f^{1} \otimes f^{1}+B(t) f^{2} \otimes f^{2}+C(t) f^{3} \otimes f^{3}, \quad t \in\left(-T_{+}, \infty\right)
$$

of the forward normalized Ricci flow (1.1). Let $\bar{g}(t)=\left(B_{0} / B(t)\right) g_{f}(t)$. Isenberg and Jackson [6] shows that $A-B \leq\left(A_{0}-B_{0}\right) e^{-4 B_{0}^{2} t}, \forall t \geq 0$, if $A_{0} \geq$ $B_{0}$ (this order is preserved by the flow). Hence, in the forward direction, $\bar{g}(t)$ converges exponentially fast to the flat metric whereas Theorem 4.1 describes the backward behavior. In the generic case $A_{0}>B_{0}, \bar{g}(t)$ converges to a sub-Riemannian metric as $t \rightarrow-T_{+}$.

In this case, the sectional curvatures are:

$$
\begin{aligned}
& K\left(f_{2} \wedge f_{3}\right)=\frac{1}{A B C}(B-A)(B+3 A) \\
& K\left(f_{3} \wedge f_{1}\right)=\frac{1}{A B C}(A-B)(A+3 B) \\
& K\left(f_{1} \wedge f_{2}\right)=\frac{1}{A B C}(A-B)^{2} .
\end{aligned}
$$

Hence the solution $g(t)=A(t) f^{1} \otimes f^{1}+B(t) f^{2} \otimes f^{2}+C(t) f^{3} \otimes f^{3}$ of the normalized positive Ricci flow satisfies

$$
\left\{\begin{array}{l}
\frac{d A}{d t}=\frac{2}{3} A(2 A+B)(A-B) \\
\frac{d B}{d t}=-\frac{2}{3} B(2 B+A)(A-B) \\
\frac{d C}{d t}=-\frac{2}{3} C(A-B)^{2}
\end{array}\right.
$$

under the normalization $A B C=4$.

If $A_{0}=B_{0}$ we clearly have $g(t)=g_{0}$ for all $t \geq 0$. Without loss of generality, we assume that $A_{0}>B_{0}$. Then $A>B$ as long as the solution exists. Hence $A$ is increasing whereas $B$ and $C$ are decreasing. 
Lemma 4.1. If $A_{0}>B_{0}$, then $T_{+}<\infty, \lim _{T_{+}} A=\infty, \lim _{T_{+}} B=\lim _{T_{+}}$ $C=0$.

Proof. Since $A-B>0$ is increasing and

$$
\frac{d}{d t}(A-B)=\frac{4}{3}(A-B)\left(A^{2}+A B+B^{2}\right)>\frac{4}{3}(A-B)^{3}
$$

we have $\frac{d}{d t}(A-B)^{-2}<-\frac{8}{3}$, so $T_{+}<\infty$.

If $\lim _{T_{+}} A=A\left(T_{+}\right)<\infty$, then

$$
\frac{d}{d t} \ln B>-2 A^{2}>-2 A\left(T_{+}\right)^{2}
$$

and

$$
\frac{d}{d t} \ln C>-\frac{2}{3} A^{2}>-\frac{2}{3} A\left(T_{+}\right)^{2}
$$

This leads to $\lim _{T_{+}} B=B\left(T_{+}\right)>0$ and $\lim _{T_{+}} C=C\left(T_{+}\right)>0$ and contradicts the fact that the maximal existence time $T_{+}$is finite.

To prove that $B$ tends to 0 , note that

$$
\frac{d}{d t} \ln \left(A B^{2}\right)=-2(A-B) B<0 .
$$

Hence $A B^{2}$ is decreasing and $\lim _{T_{+}} B=0$. Similarly,

$$
\frac{d}{d t} \ln \left(A C^{3}\right)=-\frac{2}{3}(A-B)(A-2 B)
$$

implies that $A C^{3}$ is bounded from above. Hence $\lim _{T_{+}} C=0$.

Lemma 4.2. Assume that $A_{0}>B_{0}$. Then there exist $\eta_{1}, \eta_{2} \in(0, \infty)$ such that, as t tends to $T_{+}$,

$$
A \sim \frac{\sqrt{6}}{4}\left(T_{+}-t\right)^{-1 / 2}, \quad B \sim \eta_{1}\left(T_{+}-t\right)^{1 / 4}, \quad C \sim \eta_{2}\left(T_{+}-t\right)^{1 / 4} .
$$

Proof. Since $B>0$ and $\frac{d}{d t} B \sim-\frac{2}{3} A^{2} B$, we get

$$
\int_{0}^{T_{+}} A B<A_{0}^{-1} \int_{0}^{T_{+}} A^{2} B<\infty .
$$

Observe that

$$
\frac{d}{d t} \ln \left(A B^{2}\right)=-2(A-B) B ; \frac{d}{d t} \ln \left(A C^{2}\right)=2(A-B) B .
$$


Hence (4.2) implies

$$
\lim _{T_{+}} A B^{2}>0 ; \lim _{T_{+}} A C^{2}<\infty
$$

The asymptotic behaviors of $A, B$ and $C$ now follow from $\frac{d}{d t} A \sim \frac{4}{3} A^{3}$.

This finishes the proof of Theorem 4.1.

\section{The normalized positive Ricci flow on $\operatorname{SL}(2, \mathbb{R})$}

Given a left-invariant metric $g_{0}$ on $\operatorname{SL}(2, \mathbb{R})$, we fix a Milnor frame $\left\{f_{i}\right\}_{1}^{3}$ such that

$$
\left[f_{2}, f_{3}\right]=-2 f_{1}, \quad\left[f_{3}, f_{1}\right]=2 f_{2}, \quad\left[f_{1}, f_{2}\right]=2 f_{3}
$$

and

$$
g_{0}=A_{0} f^{1} \otimes f^{1}+B_{0} f^{2} \otimes f^{2}+C_{0} f^{3} \otimes f^{3}
$$

Theorem 5.1. Let $g_{0}$ be a homogeneous metric on $\operatorname{SL}(2, \mathbb{R})$ with associated Milnor frame $\left(f_{1}, f_{2}, f_{3}\right)$ and $g_{0}=A_{0} f^{1} \otimes f^{1}+B_{0} f^{2} \otimes f^{2}+C_{0} f^{3} \otimes f^{3}$ with $A_{0} B_{0} C_{0}=4$. Let $g(t)=A(t) f^{1} \otimes f^{1}+B(t) f^{2} \otimes f^{2}+C(t) f^{3} \otimes f^{3}, t \in$ $\left[0, T_{+}\right)$be the maximal forward solution of the positive normalized Ricci flow (1.3) with $g(0)=g_{0}$. Then $T_{+}<\infty$. Assume that $B_{0} \geq C_{0}$, and set

$$
Q=\left\{(a, b, c) \in \mathbb{R}^{3}: a>0, b \geq c>0\right\}
$$

and

$$
\bar{g}(t)=\frac{C_{0}}{C(t)} g(t)
$$

There is a partition of $Q$ into subsets $S_{0}, Q_{1}, Q_{2}$ with $Q_{1}, Q_{2}$ connected such that, as tends to $T_{+}$:

(1) If $\left(A_{0}, B_{0}, C_{0}\right) \in Q_{1}$ then there exist $\eta_{1}, \eta_{2} \in(0, \infty)$ such that

$$
A \sim \frac{\sqrt{6}}{4}\left(T_{+}-t\right)^{-1 / 2}, \quad B \sim \eta_{1}\left(T_{+}-t\right)^{1 / 4}, \quad C \sim \eta_{2}\left(T_{+}-t\right)^{1 / 4}
$$

Moreover, $(M, \bar{g}(t))$ converges uniformly to the sub-Riemannian metric space $\left(M, b f_{2} \otimes f_{2}+c f_{3} \otimes f_{3}\right)$ for some $b, c \in(0, \infty)$. 
(2) If $\left(A_{0}, B_{0}, C_{0}\right) \in Q_{2}$ then there exist $\eta_{1}, \eta_{2} \in(0, \infty)$ such that

$$
A \sim \eta_{1}\left(T_{+}-t\right)^{1 / 4}, \quad B \sim \frac{\sqrt{6}}{4}\left(T_{+}-t\right)^{-1 / 2}, \quad C \sim \eta_{2}\left(T_{+}-t\right)^{1 / 4}
$$

Moreover, $(M, \bar{g}(t))$ converges uniformly to the sub-Riemannian metric space $\left(M, a f_{1} \otimes f_{1}+c f_{3} \otimes f_{3}\right)$ for some $a, c \in(0, \infty)$.

(3) If $(A(t), B(t), C(t)) \in S_{0}$ for all $t \in\left(T_{+}, 0\right]$ then

$$
A \sim \frac{\sqrt{6}}{4}\left(T_{+}-t\right)^{-1 / 2}, \quad B \sim \frac{\sqrt{6}}{4}\left(T_{+}-t\right)^{-1 / 2}, \quad C \sim \frac{32}{3}\left(T_{+}-t\right) .
$$

Remark 5.1. Cases (1) and (2) of Theorem 5.1 are somewhat symmetric. As we shall see, $Q_{1}$ contains $\{(a, b, c): a \geq b \geq c\}$ and $Q_{2}$ contains $\{(a, b, c)$ : $a \leq b-c\}$. Case (3) is of a completely different nature and it is not even entirely clear, a priori, that it occurs at all. In the forthcoming work [1], we show that $Q_{1} \cup Q_{2}$ is a dense open set in $Q$ and that $S_{0}$ is an hypersurface separating $Q_{1}$ from $Q_{2}$. This however requires different techniques than those used in this paper.

Remark 5.2. In case (3), let $d(t)$ be the metric on $M=\operatorname{SL}(2, \mathbb{R})$ induced by $g(t)$. Observe that there are no factors $r(t)$ such that $(M, r(t) d(t))$ converges uniformly to a metric structure on $M$. A meaningful scaling might be to consider $\left(M, \frac{A_{0}}{A(t)} g(t)\right)$ for which two components converge and the third goes to zero (potentially, a dimensional collapse but curvatures blow up).

Remark 5.3. For the forward normalized Ricci flow (1.1), Isenberg and Jackson [6] show that the solution exists for all time and presents a pancake degeneracy.

For the proof of Theorem 5.1, we recall that the sectional curvatures are

$$
\begin{aligned}
& K\left(f_{2} \wedge f_{3}\right)=\frac{1}{A B C}\left(-3 A^{2}+B^{2}+C^{2}-2 B C-2 A C-2 A B\right) \\
& K\left(f_{3} \wedge f_{1}\right)=\frac{1}{A B C}\left(-3 B^{2}+A^{2}+C^{2}+2 B C+2 A C-2 A B\right) \\
& K\left(f_{1} \wedge f_{2}\right)=\frac{1}{A B C}\left(-3 C^{2}+A^{2}+B^{2}+2 B C-2 A C+2 A B\right) .
\end{aligned}
$$

Therefore, writing

$$
g=A f^{1} \otimes f^{1}+B f^{2} \otimes f^{2}+C f^{3} \otimes f^{3}
$$


for the solution of the positive normalized Ricci flow with initial data $g_{0}$, $A, B, C$ (with $A B C=4$ ) satisfy the equations

$$
\left\{\begin{array}{l}
\frac{d A}{d t}=-\frac{2}{3}\left[-A^{2}(2 A+B+C)+A(B-C)^{2}\right] \\
\frac{d B}{d t}=-\frac{2}{3}\left[-B^{2}(2 B+A-C)+B(A+C)^{2}\right] \\
\frac{d C}{d t}=-\frac{2}{3}\left[-C^{2}(2 C+A-B)+C(A+B)^{2}\right] .
\end{array}\right.
$$

Without loss of generality, we may assume that $B_{0} \geq C_{0}$. Looking at the evolution equation of $B-C$, it follows that $B \geq C$ as long as a solution exists.

Since that $A B C=A_{0} B_{0} C_{0}=4$. We have

$$
\frac{d}{d t} C=-\frac{2}{3}\left[B C^{2}+B^{2} C-2 C^{3}+A B C-A C^{2}+A B C+A^{2} C\right] \leq-\frac{2}{3}
$$

$C$ is decreasing and the solution can only exist up to some finite time $T_{+}<\infty$.

Lemma 5.1. Assume that $B_{0} \geq C_{0}$, we have

$$
\lim _{T_{+}} C=0
$$

Proof. Observe that $A B$ is increasing because that

$$
\frac{d}{d t} \ln (A B)=-\frac{2}{3}\left[-B^{2}-A^{2}-2 A B-B C+A C+2 C^{2}\right]>0 .
$$

Assume that $\lim _{T_{+}} C(t)=\eta>0$. Then $A B<\frac{4}{\eta}$ and $B \geq C>\eta$. So $A<$ $\frac{4}{\eta^{2}}$ and we must have $\lim _{T_{+}} A(t)=0$ and $\lim _{T_{+}} B(t)=\infty$ (because $A B$ is increasing and bounded from above, it is easy to see those two conditions are equivalent). Hence we have

$$
\frac{d A}{d t} \sim-\frac{2}{3} A B^{2}, \quad \frac{d B}{d t} \sim \frac{4}{3} B^{3}, \quad \frac{d C}{d t} \sim-\frac{2}{3} C B^{2} .
$$

So we have $B(t)^{-2} \sim \frac{8}{3}\left(T_{+}-t\right)$, but this contradicts $\lim _{T_{+}} C(t)=\eta>0$.

Lemma 5.2. Assume $B_{0} \geq C_{0}$. If there exists a time $t_{0}$ such that $A\left(t_{0}\right) \geq$ $B\left(t_{0}\right)$ then

$$
A \sim \frac{\sqrt{6}}{4}\left(T_{+}-t\right)^{-1 / 2}, \quad B \sim \eta_{1}\left(T_{+}-t\right)^{1 / 4}, \quad C \sim \eta_{2}\left(T_{+}-t\right)^{1 / 4} .
$$


Proof. As $A B C=4$, we have $\lim _{T_{+}} A B=\infty$. Moreover,

$$
\frac{d}{d t} \ln (A / B)=2(A+B)(A+C-B) .
$$

If there exists a time $t_{0}$ such that $A\left(t_{0}\right) \geq B\left(t_{0}\right)$, then $A \geq B$ on $\left[t_{0}, T_{+}\right)$. Similarly, the condition $A>2 B$ is preserved by the flow. Assuming that $A>2 B$, we have

$$
\frac{d}{d t} B \sim-\frac{2}{3} B(A-2 B)(A+B)<0
$$

hence $\lim _{T_{+}} B(t)=B\left(T_{+}\right)<\infty$. So $\lim _{T_{+}} A(t)=\infty$ and the system 5.1 yields

$$
\left\{\begin{array}{l}
\frac{d A}{d t} \sim \frac{4}{3} A^{3} \\
\frac{d B}{d t} \sim-\frac{2}{3} B A^{2} \\
\frac{d C}{d t} \sim-\frac{2}{3} C A^{2}
\end{array}\right.
$$

This gives the desired asymptotics.

We now need to rule out the case when $B \leq A \leq 2 B$ for all $t$. In that case we have

$$
\frac{4}{3} A^{3} \leq \frac{d A}{d t} \leq 2 A^{3}
$$

this implies $\int_{0}^{T_{+}} A^{2}=\infty$. Further, by $(5.2), \int_{0}^{T_{+}}\left(A^{2}-B^{2}\right)<\infty$. Since $A / B$ is non-decreasing, there exists a constant $\eta$, such that $A-B>\eta A$. Thus $\int_{0}^{T_{+}} A^{2}<\infty$. This is a contradiction.

Lemma 5.3. Assume $B_{0} \geq C_{0}$. If there exists a time $t_{0}$ such that $A\left(t_{0}\right) \leq$ $B\left(t_{0}\right)-C\left(t_{0}\right)$ then

$$
A \sim \eta_{1}\left(T_{+}-t\right)^{1 / 4}, \quad B \sim \frac{\sqrt{6}}{4}\left(T_{+}-t\right)^{-1 / 2}, \quad C \sim \eta_{2}\left(T_{+}-t\right)^{1 / 4} .
$$

Proof. We have

$$
\frac{d}{d t}(B-A-C)=-\frac{2}{3}\left(2 A^{3}+2 C^{3}-2 B^{3}+2 A^{2} B+2 A B C-2 A B^{2}-2 A^{2} C\right) .
$$

Hence the condition $B-A \geq C$ is preserved by the flow. It follows from the flow equations (5.1) and (5.2) that both $B$ and $B / A$ are increasing. 
So we have $\lim _{T_{+}} B=\infty$. If $\lim _{T_{+}} B / A<\infty$ then, since there exists a $\eta$ such that $B-A-C>\eta B,(5.2)$ yields that $\int_{0}^{T_{+}} B^{2}<\infty$. The evolution equation (5.1) shows that this contradicts $\lim _{T_{+}} B(t)=\infty$. Hence we must have $\lim _{T_{+}} B / A=\infty$, and (5.1) gives

$$
\left\{\begin{array}{l}
\frac{d A}{d t} \sim-\frac{2}{3} A B^{2} \\
\frac{d B}{d t} \sim \frac{4}{3} B^{3} \\
\frac{d C}{d t} \sim-\frac{2}{3} C B^{2} .
\end{array}\right.
$$

This proves the desired result.

Now the only case left is when $A<B<A+C$ for all $t \in\left[0, T_{+}\right)$. In this case since $\lim _{T_{+}} C=0$, we have $\lim _{T_{+}}(B-A)=0$, and the flow equation (5.1) yields that

$$
\left\{\begin{array}{l}
\frac{d A}{d t} \sim \frac{4}{3} A^{3} \\
\frac{d B}{d t} \sim \frac{4}{3} B^{3} \\
\frac{d C}{d t} \sim-\frac{8}{3} C B^{2} .
\end{array}\right.
$$

So we arrive at

$$
A \sim \frac{\sqrt{6}}{4}\left(T_{+}-t\right)^{-1 / 2}, \quad B \sim \frac{\sqrt{6}}{4}\left(T_{+}-t\right)^{-1 / 2}, C \sim \frac{32}{3}\left(T_{+}-t\right) .
$$

This together with the two previous lemmas concludes the proof of Theorem 5.1. As noted in the remark following the theorem, it is not clear from the proof itself that the third case does indeed occur. In [1], we show that there is a smooth hypersurface of initial condition which is preserved by the flow, which exactly corresponds to the asymptotic behavior described in the third case $A<B<A+C$ for all $t \in\left[0, T_{+}\right)$.

\section{Acknowledgments}

Research of X.C. was partially supported by the Jeffrey Sean Lehman Fund from Cornell University and the Research of L.S.-C. was partially supported by NSF grant DMS 0603866 . 


\section{References}

[1] X. Cao, J. Guckenheimer and L. Saloff-Coste, The backward behavior of the Ricci and cross curvature flows on $S L(2, R), 2008$, Preprint.

[2] B. Chow and D. Knopf, The Ricci flow: an introduction, Mathematical Surveys and Monographs, 110, American Mathematical Society, Providence, RI, 2004.

[3] X. Cao, Y. Ni and L. Saloff-Coste, Cross curvature flow on locally homogeneous three-manifolds. I, Pacific J. Math. 236(2) (2008), 263-281.

[4] X. Cao and L. Saloff-Coste, The cross curvature flow on locally homogeneous three-manifolds (ii), 2008, arXiv:0805.3380v1 [math.DG].

[5] D. Glickenstein, Riemannian groupoids and solitons for threedimensional homogeneous Ricci and cross-curvature flows, Int. Math. Res. Not. IMRN, no. 12 (2008), Art. ID rnn034, 49pp.

[6] J. Isenberg and M. Jackson, Ricci flow of locally homogeneous geometries on closed manifolds, J. Differential Geom. 35(3) (1992), 723-741.

[7] D. Knopf and K. McLeod, Quasi-convergence of model geometries under the Ricci flow, Comm. Anal. Geom. 9(4) (2001), 879-919.

[8] J. Lott, On the long-time behavior of type-iii Ricci flow solutions, Math. Ann. 339(3) (2007), 627-666.

[9] J. Milnor, Curvatures of left invariant metrics on Lie groups, Adv. Math. 21(3) (1976), 293-329.

[10] R. Montgomery, A tour of subriemannian geometries, their geodesics and applications, Mathematical Surveys and Monographs, 91, American Mathematical Society, Providence, RI, 2002.

Department of Mathematics

CORNELL UNIVERSITY

ITHACA, NY 14853

USA

E-mail address: cao@math.cornell.edu

E-mail address: lsc@math.cornell.edu

ReCEIVED OCTOBER 24, 2008 
